\title{
Current perspectives in intronic micro RNAs (miRNAs)
}

\author{
Shao-Yao Ying \& Shi-Lung Lin \\ Department of Cell \& Neurobiology, Keck School of Medicine, BMT-403, University of Southern California, \\ 1333 San Pablo Street, Los Angeles, CA, 90033, USA
}

Received 27 May 2005; accepted 14 September 2005

(c) 2005 National Science Council, Taipei

Key words: fine-tuning of gene function, functional/structural genomics, gene expression, genetic regulation, intronic microRNA, miRNA biogenesis, miRNA, post-translational modification, regulatory gene

\begin{abstract}
Summary
MicroRNAs (miRNAs), small single-stranded regulatory RNAs capable of interfering with intracellular messenger RNAs (mRNAs) that contain either complete or partial complementarity, are useful for the design of new therapies against cancer polymorphism and viral mutation. Numerous miRNAs have been reported to induce RNA interference (RNAi), a post-transcriptional gene silencing mechanism. Intronic miRNAs, derived from introns by RNA splicing and Dicer processing, can interfere with intracellular mRNAs to silence that gene expression. The intronic miRNAs differ uniquely from previously described intergenic miRNAs in the requirement of type II RNA polymerases (Pol-II) and spliceosomal components for its biogenesis. Several kinds of intronic miRNAs have been identified in Caenorhabditis elegans, mouse and human cells; however, neither their function nor application has been reported. To this day, the computer searching program for miRNA seldom include the intronic portion of protein-coding RNAs. The functional significance of artificially generated intronic miRNAs has been successfully ascertained in several biological systems such as zebrafishes, chicken embryos and adult mice, indicating the evolutionary preservation of this gene regulation system in vivo. Multiple miRNAs can be generated from the same cluster of introns; however, non-homologous miRNAs may have different targets and functions while homologous miRNA may be derived from different intronic clusters. Taken together, the model of intronic miRNAmediated transgenic animals provides a tool to investigate the mechanism of miRNA-associated diseases in vivo and will shed light on miRNA-related therapies.
\end{abstract}

\section{Introduction}

The intronic microRNA (miRNA) is a form of gene that regulates the function of other genes. It probably plays a critical role as gene regulators in fine-tuning many biological manifestations. The gene is conventionally defined as the specific region of a DNA molecule that codes for the synthesis of a particular protein. This specific region consists of exons and introns; exons code the protein sequences

*To whom correspondence should be addressed. Fax: +323442-3466; E-mail: sying@usc.edu whereas introns are thought to be junk genes. We know now that this perception needs to be modified.

The Human Genome Project has completed the process of determining, sequencing, and mapping more than 36,000 genes and about 3 billion nucleotides bases of the human genome. What dose the vast majority of the nucleotides do in our body? The purpose of this article is to provide an up-to-date summary of new knowledge that is directly resulted from the studying of the intronic miRNA as an example to illustrate the role of the so-called "junk genes." 
The intronic miRNA is defined as small RNAs, derived from introns, processed by splicing and pre-miRNA degradations and targets the intracellular mRNA for silencing of that gene expression. The intron occupies the largest proportion of noncoding sequences in the protein-coding DNA of a genome. The transcription of the genomic proteincoding DNA generates precursor messenger RNA (pre-mRNA).

The pre-mRNA consists four major parts: the $5^{\prime}$-untranslational region (UTR), the proteincoding exon, the non-coding intron and the 3'-UTR region. Both the 5'- and 3'-UTR regions can be broadly defined as a kind of intron extension, however, the processing of these two regions during mRNA translation is different from the intron located between two protein-coding exons, termed the in-frame intron. The in-frame intron can be as big as several $10 \mathrm{k}$-nucleotides and was thought to be a huge genetic waste in gene transcripts. Recently, the discovery of the intronderived microRNA (intronic miRNA) completely changed this perception.

The intronic miRNA is a new class of small single-stranded regulatory RNAs and is different from previously identified miRNAs. First, the intronic miRNA is derived from the processing of pre-mRNA introns. Second, it requires unique Pol-II and RNA splicing components for its biogenesis [1-3]. Third, about $10 \sim 30 \%$ of the spliced intron are exported out of the nucleus and have a moderate half-life in the cytoplasm [4]. The messenger RNA (mRNA) is usually 18-25 oligonucleotides capable of either directly degrading its intracellular mRNA target or suppressing the protein translation of its targeted mRNA, depending on the complementarity between the miRNA and its target. In this sense, the intronic miRNA is similar to previously described intergenic miRNAs structurally and functionally. Good examples of intronic miRNA have been described in Caenorhabditis elegans, mouse and human genomes [2, 5, 6] and some of their functions are closely related to RNA interference (RNAi).

\section{$R N A$ interference ( $R N A i)$}

RNA interference is a post-transcriptional gene silencing mechanism in eukaryotes, which can be triggered by small RNA molecules such as miRNA and small interfering RNA (siRNA). These two types of small RNA molecules usually interfere with expression of genes intracellularly based on their completely or partially complementary of the small RNAs to the target gene, respectively. Basically, siRNAs are double-stranded RNAs that degrade target gene transcripts based on nearly perfect complementarity between these two RNA molecules [7, 8]. miRNAs, unlike the stringent complementarity of siRNAs to their RNA targets, are single-stranded that pair with target RNAs, which have partial complementarity to the miRNAs [9, 10]. Many native miRNAs have been located in hairpin-like RNA precursors in eukaryotes, including yeast (Schizosaccharomyces pombe), plant (Arabidopsis spp.), nematode ( $C$. elegans), fly (Drosophila melanogaster), mouse, and human. These native miRNAs are usually involved in defense against viral infections and regulation of gene expression during development [11-21]. In contrast, native siRNAs are mainly demonstrated in plants and low-level animals (worms and flies) and their prevalence in mammals remains to be established [10]. Due to the fact that miRNAs are widely detected in eukaryotes, these small RNAs have recently been used to design reagents against cancers and viral infections as a novel gene therapy $[1,22]$. Indeed, gene silencing mechanisms involving miRNAs have been proposed to be an intracellular defense system as a means to eliminate undesired transgenes and foreign RNAs, including viral infections and retrotransposon activities [22, 23].

\section{miRNA biogenesis}

The process of miRNA biogenesis in vertebrates is presumably involved five steps (Figure 1). First, miRNA is generated as a long primary precursor miRNA (pri-miRNA) which is most likely mediated by RNA polymerases type II (Pol-II) [2, 24]. Second, the long pri-miRNA is excised by Droshalike RNase III endonucleases and/or spliceosomal components to form the precursor miRNA (premiRNA). This step is depending on the origin of the pri-miRNA located in an exon or an intron respectively [2, 25]. Third, the pre-miRNA is exported out of the nucleus by Ran-GTP and a receptor Exportin-5 [26, 27]. In the cytoplasm, Dicer-like nucleases cleave the pre-miRNA to form mature miRNA. Lastly, the mature miRNA is incorporated into a ribonuclear particle (RNP) 


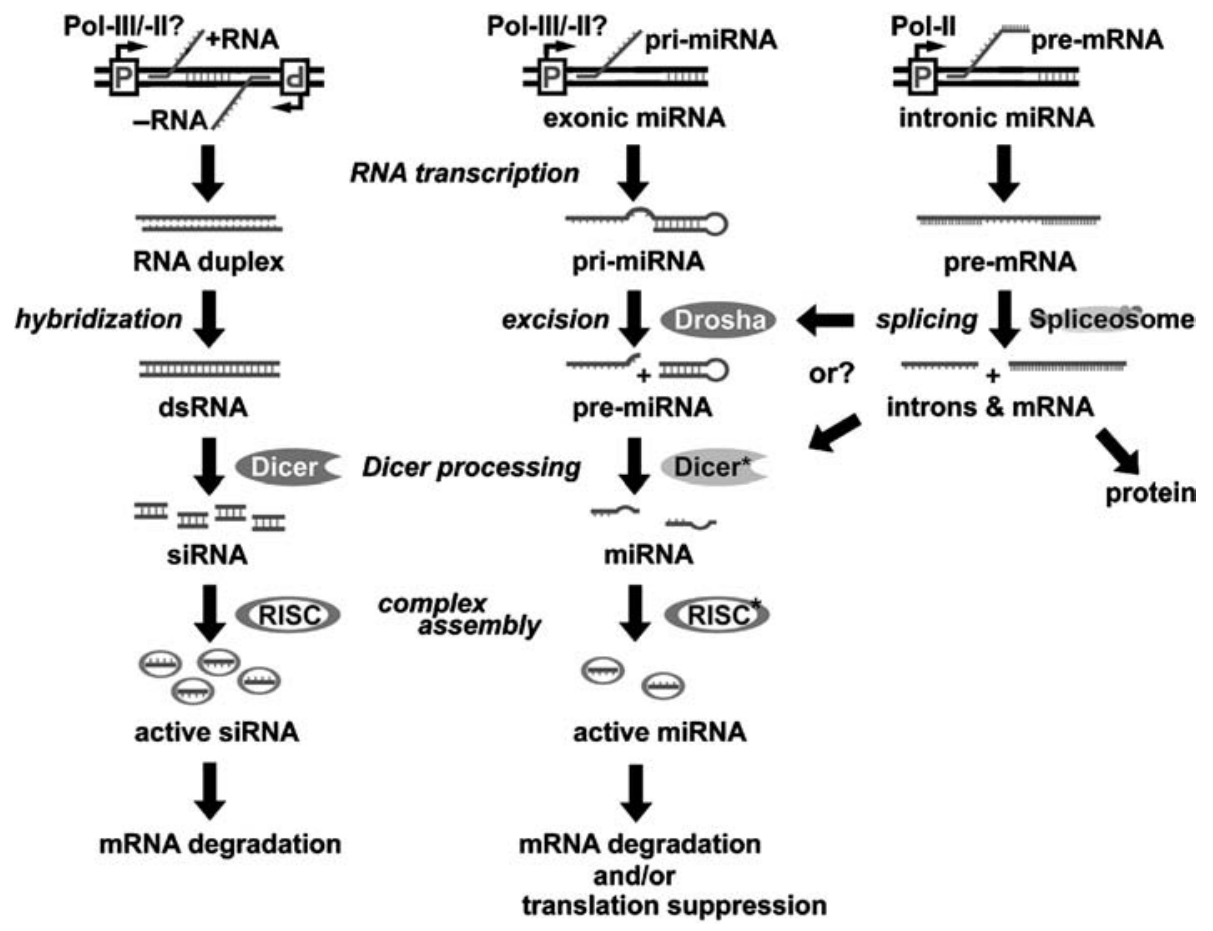

Figure 1. Comparison of biogenesis and RNAi mechanisms among siRNA, intergenic (exonic) miRNA and intronic miRNA. SiRNA is likely formed by two perfectly complementary RNAs transcribed from two different promoters (remains to be determined) and further processing into 19-22 bp duplexes by RNase III-familial endonuclease, Dicer. The biogenesis of intergenic miRNA, e.g. lin-4 and let-7, involves a long transcript precursor (pri-miRNA), which is probably generated by a Pol-II or Pol-III RNA promoter, while intronic miRNA is transcribed by the Pol-II promoter of its encoded gene and co-expressed in the intron region of the gene transcript (pre-mRNA). After RNA splicing and further processing, the spliced intron may function as a pri-miRNA for intronic miRNA generation. In the nucleus, the pri-miRNA is excised by Drosha-like RNases to form a hairpin-like pre-miRNA template and then exported to cytoplasm for further processing by Dicer* to form mature miRNAs. The asterisk * indicates that the Dicers for siRNA and miRNA pathways are different. All three small regulatory RNAs are finally incorporated into a RNAinduced silencing complex (RISC), which contains either strand of siRNA or the single-strand of miRNA. The effect of miRNA is considered to be more specific and less adverse than that of siRNA because only one strand is involved. On the other hand, siRNA primarily triggers mRNA degradation, whereas miRNA can induce either mRNA degradation or suppression of protein synthesis depending on the sequence complementarity to the target gene transcript.

to form the RNA-induced gene silencing complex (RISC) which executes the RNAi-related gene silencing effects [28, 29]. Although the in vitro model of siRNA-associated RISC assembly has been reported, the link between the final miRNA maturation and RISC assembly remains to be determined. The characteristics of Dicer and RISC in the siRNA and miRNA processing have been reported to be distinct [30]. In zebrafish, we have recently observed that the stem-loop structure of pre-miRNAs is involved in the strand selection for mature miRNA during RISC assembly. These findings suggest that the duplex structure of siRNA may not be required for the assembly of miRNA-associated RISC in vivo. The current biogenesis of miRNA and siRNA seems to be similar, however, the mechanisms by which
miRNA is formed as proposed by several laboratories were based on the siRNA model, therefore, one must distinguish the individual properties and differences of Dicer and RISC in these two types of small RNAs processing, particularly for the understanding evolutional and functional relationship between these two gene silencing pathways. In addition, the differences between these two gene silencing pathways may provide a clue for the prevalence of native siRNAs in invertebrates but rarely in mammals.

\section{Definition of intronic miRNAs}

The characteristics of intronic miRNA are determined by two events during biogenesis; first, they must share the same promoter with their 
encoded genes, and second, they are spliced out of the transcript of their encoded genes and further processed into mature miRNAs. Although some of currently identified miRNAs are encoded in the genomic intron region of a gene that have an orientation opposite to the gene transcript, they are not intronic miRNAs because they neither share the same promoter with the gene nor need to be released from the gene transcript by RNA splicing. For the transcription of those miRNAs, their promoters are located in the antisense direction to the gene, likely using the gene as a potential target for these antisense miRNAs. A good example is let-7c, which was found to be an intergenic miRNA located in the antisense region of the intron of a gene. Current computer programs for miRNA prediction usually include these intergenic miRNAs but not the intronic miRNAs. Because the intronic miRNAs are encoded in the gene transcript precursors (pre-mRNAs) and share the same promoter with the encoded gene transcripts, the current miRNA programs tend to classify intronic miRNAs as protein-coding regions and are excluded as miRNAs in the prediction. Thus, the miRNA-prediction program including database of non-coding sequences in the protein-coding premRNA regions is urgently needed for thoroughly screening the hairpin-like intronic miRNAs in the genomes.

\section{miRNAs and human diseases}

The majority of human gene transcripts contain introns, which vary from each other. Changes in these non-protein-coding sequences frequently results in clinical and circumstantial malfunction. Numerous introns were recently identified to encode miRNAs, which are involved in RNAirelated chromatin silencing mechanisms. Over 90 intronic miRNAs have been identified using the bioinformatics approaches to date, but the function of the vast majority of these identified molecules remains to be determined [6]. Conceivably, condition-specific, time-specific, and individual-specific fine-tuning levels of gene expression can be considered as the interaction of different miRNAs on single or multiple gene modulation. According to the strictly expressive correlation of intronic miRNAs to their target genes, the interaction between miRNAs and target genes more accurately accounts for genetic expression of various traits and, therefore dysregulation of this interaction will result in genetic diseases.

For instance, monozygotic twins frequently demonstrate slightly, but definitely distinguishing, disease susceptibility and physiological behavior. A long CCTG expansion in the intron 1 in a zinc finger protein ZNF9 gene has been correlated to the type 2 myotonic dystrophy in the twin with higher susceptibility [31]. Given that the expansion motif has high affinity to certain RNA-binding proteins, the interfering role of intron-derived expansion fragments remains to be elucidated.

Another example is the fragile $\mathrm{X}$ syndrome, a disease represents about $30 \%$ of human inherited mental retardation, which involves intronic expansion fragments of the FMR1 gene in the pathogenesis of this syndrome. The intronic CGG repeat (rCGG) expansion in the $5^{\prime}$-UTR of FMRl gene is the causative mutation in $99 \%$ of individuals with fragile X syndrome [32]. FMRI encodes an RNAbinding protein, FMRP, which is associated with polyribosome assembly in an RNP-dependent manner and suppresses translation through an RNAi-like pathway. FMRP also contains a nuclear localization signal (NLS) and a nuclear export signal (NES) for shuttling certain mRNAs between the nucleus and cytoplasm [33]. Jin et al. proposed an RNAi-mediated methylation model in the $\mathrm{CpG}$ region of FMR1 rCGG expansion, which is targeted by a hairpin RNA derived from the $3^{\prime}$-UTR of the FMRI expanded allele transcript [32]. The Dicer-processed hairpin RNA triggers the formation of RNA-induced initiator of transcriptional gene silencing (RITS) on the homologous rCGG sequences and leads to heterochromatin repression of the FMRI locus.

Given that natural evolution give raise to more complexity and more variety of introns in higher animals and plants for coordinating the volumes and interactions of the vast gene expression, the dysregulation of intronic miRNAs may provide a fundamental clue for the genetic diseases involving intronic expansion or deletion, such as myotonic dystrophy and fragile $\mathrm{X}$ mental retardation. In this way, gene expression produces not only gene transcripts for its own protein synthesis but also intronic miRNAs, capable of interfering with other gene expressions. According to this concept, the expression of a gene encoding a protein results in the gain-of-function of exons of the gene. And, at the same time, the loss-of-function of some 
other genes owing to the mature intronic miRNAs showing complementarity to these other genes. Thus, an array of genes can swiftly and accurately coordinate their expression patterns with each other through the mediation of their intronic miRNAs, bypassing the time-consuming translation processes under quickly changing environments. Conceivably, intron-mediated gene regulation may be as important as the mechanisms by which transcription factors regulate gene expression. It is likely that intronic miRNA is able to trigger cell transitions in response to external stimuli without the tedious protein synthesis. Undesired gene products are suppressed by both transcriptional inhibition and/or translational suppression mediated through miRNAs. This could enable a rapid switch to a new gene expression pattern without the need to produce various transcription factors. This regulatory property of miRNA may be one of the most ancient gene modulation systems before the emergence of protein. According to the variety of miRNAs and the complexity of genomic introns, a thorough investigation of miRNA variants in human genome will markedly improve the understanding of genetic diseases and also the design of miRNA-based drugs for future gene therapies.

\section{Advantages of miRNA-mediated RNAi mechanism}

To understand the disease caused by dysregulation of intronic miRNA, an artificial expression system is needed to recreate the function and mechanism of the miRNA in vitro and in vivo. The same approach may be used to design and develop therapies for the disease. Previously, several vector-based RNAi expression systems have been developed, using type-III RNA polymerase (PolIII)-directed transcription activities, to generate more stable RNAi efficacy and lower interferonrelated toxicity in several cell lines in vitro [34-37], as well as mice in vivo [38, 39]. However, their delivery strategies did not target a specific cell population due to the ubiquitous existence of PolIII activity in all cell types. Because the readthrough side-effect of Pol-III occurs on a short transcription template if without proper termination, large RNA products longer than desired 1825 base-pairs (bp) can be synthesized and then cause unexpected interferon cytotoxicity [40, 41]. Such a problem can also result from the competitive conflict between the Pol-III promoter and another vector promoter (i.e. LTR and CMV promoters).

We and others have found that high dosage of siRNAs (e.g., >250 nM in human T cells) is able to cause strong cytotoxicity similar to that of long double-stranded dsRNAs [42, 43]. This toxicity is due to the double-stranded structure of siRNAs and dsRNAs, which activates the interferon-mediated non-specific RNA degradation and programmed cell death through the signaling of PKR and 2-5A systems. It is well known that interferon-induced protein kinase PKR can trigger cell apoptosis, while activation of interferoninduced $2^{\prime}, 5^{\prime}$-oligoadenylate synthetase (2-5A) system leads to extensive cleavage of single-stranded RNAs (i.e. mRNAs) [44]. Both PKR and 2-5A systems contain dsRNA-binding motifs which are highly conserved for binding to dsRNAs, but these motifs do not bind to either single-strand RNAs or RNA-DNA hybrids. Therefore, the use of miRNA may offer alternative advantages to improve the siRNA drawbacks.

Intron-derived miRNA system is able to be activated in a specific cell type under the control of type-II RNA polymerases (Pol-II)-directed transcriptional machinery. To overcome the Pol-IIImediated siRNA side-effects, we have successfully developed a novel Pol-II-based miRNA biogenesis strategy, employing intronic miRNA molecules [2] to knock down more than $85 \%$ of selected oncogene function or viral genome replication $[45,46]$. Because of the flexibility in binding with partially complementary mRNA targets, miRNA can serve as an anti-cancer drug or vaccine to achieve a major breakthrough in the treatments of cancer polymorphisms and viral mutations. We are the first research group who discovered the biogenesis of miRNA-like precursors from the $5^{\prime}$-proximal intron regions of gene transcripts (premRNAs) produced by the mammalian Pol-II. Depending on the promoter of the miRNAencoded gene transcript, intronic miRNA is co-expressed with its encoding gene in the specific cell population, which activates the promoter and expresses the gene.

Some spliced introns are not completely digested into monoribonucleotides for transcriptional recycling since approximately $10-30 \%$ of the intron fragments cam be found in the cytoplasm with a moderate half-life $[4,47]$. This type of 
miRNA generation has been reported to rely on the coupled interaction of nascent Pol-II-mediated pre-mRNA transcription and intron excision, occurring within certain nuclear regions proximal to genomic perichromatin fibrils [46, 48, 49]. After Pol-II RNA processing and splicing excision, some of the intron-derived hairpin RNA fragments can form mature miRNAs and effectively silence the target gene through the RNAi mechanism, while the exons of pre-mRNA are ligated together to form a mature mRNA for protein synthesis (Figure 2A) [2]. Since miRNAs are single-stranded molecules insensitive to PKR- and 2-5A-induced interferon systems, the utilization of this Pol-II-mediated miRNA generation can be safe in vitro and in vivo without the cytotoxic effects of dsRNAs and siRNAs. These findings indicate new functions for mammalian introns in intracellular
miRNA generation and gene silencing, which can be used as a tool for analysis of gene functions and development of gene-specific therapeutics against cancers and viral infections.

\section{Generation of man-made intronic miRNAs}

Using man-made introns carrying hairpin-like miRNA precursors (pre-miRNA), we have successfully generated mature miRNA molecules with full capacity in triggering RNAi-like gene silencing in human prostate cancer LNCaP, human cervical cancer HeLa and rat neuronal stem HCN-A94-2 cells $[2,45]$. As shown in Figure 2B, the artificial intron (SpRNAi) is co-transcribed within a premRNA by Pol-II and cleaved out of the premRNA by RNA splicing. Then, the spliced intron containing a pre-miRNA structure is further

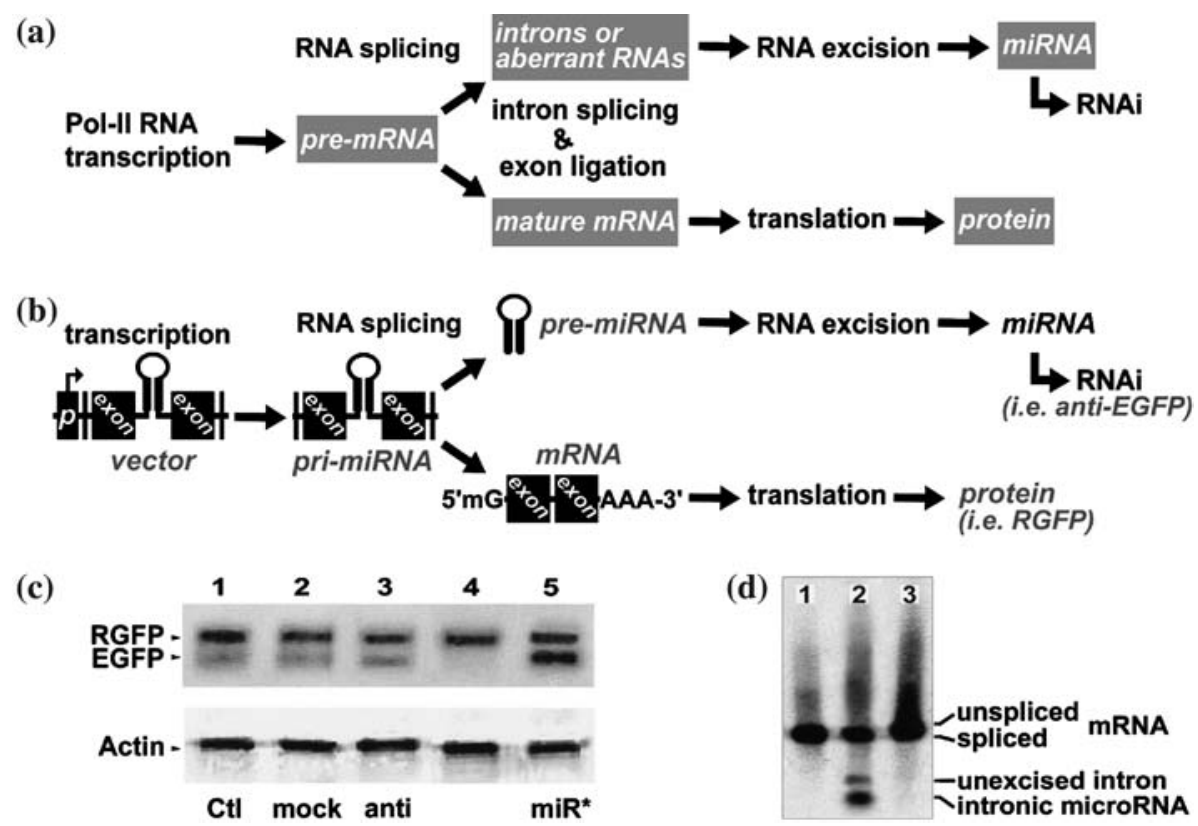

Figure 2. Biogenesis and function of intronic miRNAs. (A) The native intronic miRNA is co-transcribed with a precursor messenger RNA (pre-mRNA) by Pol-II and cleaved out of the pre-mRNA by an RNA splicing machinery, spliceosome. The spliced intron with hairpin-like secondary structures is further processed into mature miRNAs capable of triggering RNAi effects, while the ligated exons become a mature messenger RNA (mRNA) for protein synthesis. (B) We designed an artificial intron containing a pre-miRNA strucure, namely SpRNAi, mimicking the biogenesis processes of the native intronic miRNAs. (C) When a designed miR-EGFP(280-302)-stemloop RNA construct was tested in the EGFP-expressing Tg(UAS:gfp) zebrafishes, we detected a strong RNAi effect only on the target EGFP (lane 4). No detectable gene silencing effect was observed in other lanes from left to right: 1, blank vector control (Ctl); 2, miRNA-stemloop targeting HIV-p24 (mock); 3, miRNA without stemloop (anti); and 5, stemloopmiRNA* complementary to the miR-EGFP(280-302) sequence (miR*). The off-target genes such as vector RGFP and fish actin were not affected, indicating the high target specificity of miRNA-mediated gene silencing. (D) Three different miR-EGFP(280-302) expression systems were tested for miRNA biogenesis from left to right: 1, vector expressing an intron-free gene, no pre-miRNA insert; 2, vector expressing a gene with intronic pre-miRNA insertion; and 3, vector similar to the 2 construct but with a defected 5 '-splice site in the intron. Northern bolt analysis showed that the mature miRNA was released only from the spliced intron resulted from the vector 2 construct in the cell cytoplasm. 
processed into mature miRNAs capable of triggering RNAi-related gene silencing effects. Based on this artificial miRNA model, we have tested various pre-miRNA constructs, and observed that the production of intron-derived miRNA fragments was originated from the 5 '-proximity of the intron sequence between the $5^{\prime}$-splice site and the branching point (Figure 3 ).

These miRNAs are able to trigger strong suppression of genes possessing more than 70\% of complementarity to the miRNA sequence, whereas non-homologous miRNAs, i.e. empty intron without the pre-miRNA insert, intron with an off-target miRNA insert (negative control), and splicing-defective intron have no silencing effects on the targeted gene. The same results can also be reproduced in the zebrafish directed against target EGFP expression (Figure 2C), indicating the consistent preservation of the intronic miRNA biogenesis system in vertebrates. Further, no effect was detected on off-target genes, such as RGFP and $\beta$-actin, suggesting the high specificity of miRNA-directed RNAi.

We have confirmed the identity of the intronderived miRNAs, which are sized about 18-25 base nucleotides (nt), approximately similar to the newly identified intronic miRNAs in C. elegans. Moreover, the intronic small RNAs isolated by guanidinium-chloride ultracentrifugation can elicit strong, but short-term gene silencing effects on the homologous genes in transfected cells, indicating their temporary RNAi effects. Thus, the long-term ( $>$ month) gene silencing effect that we observed in vivo, using the Pol-II-mediated intronic miRNA system, is likely maintained by constitutively
miRNA production from the vector rather than the stability of the miRNAs.

\section{Intron-mediated gene silencing in zebrafishes}

Intronic miRNAs can be used as an effective strategy to silence specific target gene in vivo. We have resolved the structural design of pre-miRNA inserts for the best gene silencing effect in vivo and found out that a strong structural bias exists in the selection of a mature miRNA strand during assembly of the RNAi effector, RISC. RISC is a protein-RNA complex that directs either target gene transcript degradation or translational repression through the RNAi mechanism. Formation of siRNA duplexes has been reported to play a key role in assembly of the siRNA-associated RISC. The two strands of the siRNA duplex are functionally asymmetric, but assembly into the RISC complex is preferential for only one strand. Such preference is determined by the thermodynamic stability of each 5 '-end base-pairing in the strand. Based on this siRNA model, the formation of miRNA and its complementary miRNA (miR$\mathrm{NA}^{*}$ ) duplexes was thought to be an essential step for the assembly of miRNA-associated RISC. If this were true, no functional bias would be observed in the stemloop of a pre-miRNA. Nevertheless, we observed that the stemloop of the intronic pre-miRNA was involved in the strand selection of a mature miRNA for RISC assembly in zebrafish.

In these experiments, we constructed miRNAexpressing SpRNAi-RGFP vectors as previously described [2] and two symmetric pre-miRNAs,

\section{Pre-mRNA construct with SpRNAi:}

\begin{tabular}{|c|c|}
\hline -promoter & exon 1 - artificial intron (SpRNAi) - exon 2 3' T codons \\
\hline splice & $\mathrm{BrP}$ \\
\hline
\end{tabular}

After intronic insert spliced: 5'-UTR exon 1-exon 2(mRNA) 3'-UTR

+ Intronic microRNAs

Figure 3. Schematic construct of the artificial SpRNAi intron in a recombinant gene SpRNAi-RGFP for intracellular expression and processing. The components of the Pol-II-mediated SpRNAi system include several consensus nucleotide elements consisting of a $5^{\prime}$-splice site, a branch-point domain (BrP), a poly-pyrimidine tract (PPT), a 3'-splice site and a pre-miRNA insert located between the $5^{\prime}$-splice site and the BrP domain. The expression of the recombinant gene is under the regulation of either a mammalian Pol-II RNA promoter or a compatible viral promoter for cell-type-specific effectiveness. Mature miRNAs are released from the intron by RNA splicing and further Dicer processing. 
sense-stemloop-antisense (1) and antisense-stemloop-sense (2), were synthesized and inserted into the vectors, respectively. Both pre-miRNAs contained the same complementary stem arm region, which was directed against target EGFP. Because the intronic insert region of the SpRNAi-RGFP vector is flanked with a $P v u I$ and an MluI restriction site at the $5^{\prime}$ - and $3^{\prime}$-ends, respectively, the primary insert can be easily removed and replaced by various gene-specific inserts (e.g. antiEGFP) possessing cohesive ends. By changing the pre-miRNA inserts directed against different gene transcripts, this intronic miRNA generation system provides a valuable tool for genetic and miRNA-associated research in vivo.

To determine the structural preference of the designed pre-miRNAs, we have isolated all potential miRNAs complementary to the target EGFP region in the $\mathrm{Tg}$ (UAS:gfp) zebrafish (Gene, in press). The EGFP expression was constitutively driven by a $\beta$-actin promoter located in almost all cell types of the zebrafish. One effective miRNA identity, miR-EGFP(280-302), was verified in the transfections of the antisense-stemloop-sense (2) construct, as shown in the Figure 4A (gray-shading sequences). Figure 4B further shows that transfection of the SpRNAi-RGFP vector into the Tg(UAS:gfp) zebrafish co-expressed the red fluorescent protein RGFP, serving as a positive indicator for the miRNA generation in the transfected cells.

This approach has been successfully used in several mouse and human cell lines to show the RNAi effects [2, 45]. We applied the same procedure to the fishes and found that the vector easily penetrated almost all tissues of the 2-week-old zebrafish larvae within $24 \mathrm{~h}$, reaching systemic delivery of the miRNA effect. The indicator RGFP was detected in both of the fishes transfected by either pre-miRNA, whereas the silencing of target EGFP expression (green) was observed only in the fish transfected by the antisense-stemloop-sense (2) pre-miRNA (Figure 4B and C). The suppression level in the gastrointestinal (GI) tract was found to be less effective, probably due to the high RNase activity in this region. Because thermostability in the ends of the sense-antisense stem arm duplexes from both pre-miRNA constructs are the same, we suggest that the stemloop of pre-miRNA is involved in strand selection of mature miRNA during RISC assembly. Given that the cleavage site of Dicer in the stem arm determines the strand selection of mature miRNA [25], the stemloop may function as a determinant for the recognition of a special cleavage site. Therefore, the different stemloop structures among various species may also provide a clue for the prevalence of native siRNAs in invertebrates but rarely in mammals.

\section{Intron-mediated gene silencing in adult mice}

We have successfully tested the feasibility of localized gene silencing in vivo by the intronic miRNA approach and also discovered that the interaction between pre-mRNA and genomic DNA may be essential for the miRNA biogenesis [50]. We used the vector-based miRNA delivery approach in mice. Patched albino (white) skins of melanin-knockout mice (W-9 black) were created by a succession of intra-cutaneous (i.c.) transduction of about anti-tyrosinase (Tyr) pre-miRNA construct $(50 \mu \mathrm{g})$ for 4 days (total $200 \mu \mathrm{g}$ ). The Tyr catalyzes the critical and rate-limiting step of tyrosine hydroxylation in the biosynthesis of melanin in the skin and hairs. The miRNA against tyrosinase blocked the expression of melanin and Northern blot analysis showed the reduction of Tyr expression (data not shown). These findings show that utilization of intronic miRNA vectors provides a powerful new strategy for in-vivo gene therapy, potentially to melanoma, underscoring that the miRNA is effective in vivo and has no sideeffects of dsRNA.

\section{Conclusion}

The intronic miRNA represents a new frontier in genetics research. With the completion of genomesequencing projects, a major challenge will be to understand gene function and regulation. Achieving this goal will require determining how intronic miRNAs co-transcribed with exons are imposed on gene expressions. The various gene-silencing mechanisms based on complete and/or partial complementarity and their intertwined actions are beginning to reveal a fine-tuning circuit of controls that can be used to modify gene expression at the post-transcriptional level and RNA turnover. The evidence of intronic miRNA-induced silencing of gene expression in cell lines, zebra fishes, chicken embryos and local mouse 
(a)



(b)

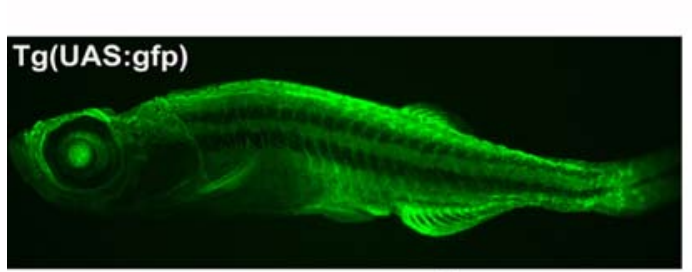

(c)
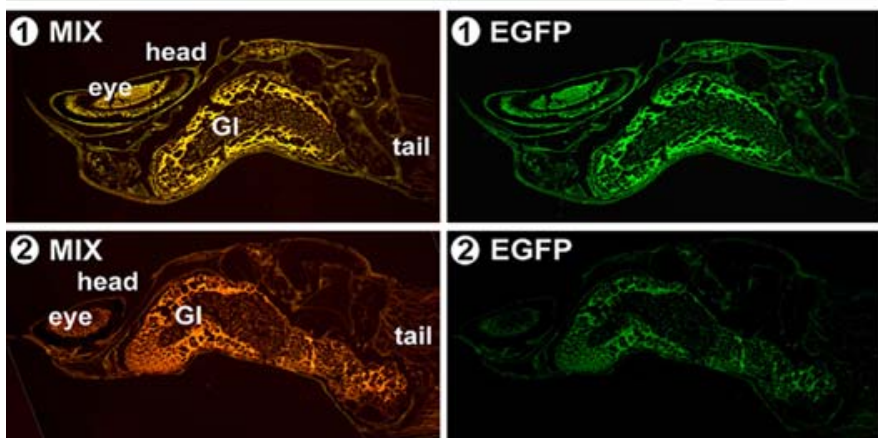

pre-miRNA insert

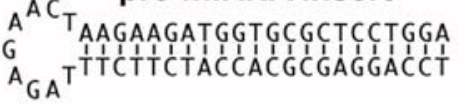

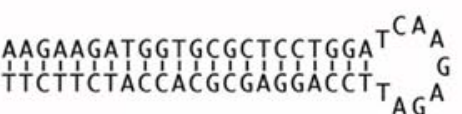

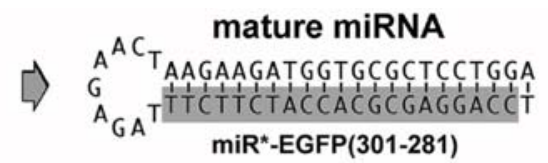

miR*-EGFP(301-281)

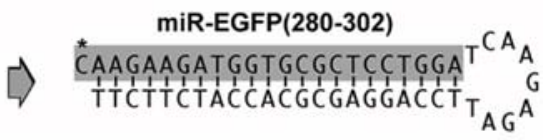

Figure 4. Bias of miRNA-miRNA* asymmetry in RISC in vivo. Different preferences of RISC assembly were observed by transfection of sense-stemloop-antisense (1) and antisense-stemloop-sense (2) pre-miRNA constructs in zebrafish, respectively. (A) Based on the assembly rule of siRNA RISC, the processing of both (1) and (2) pre-miRNAs should result in the same siRNA duplex for RISC assembly; however, the experiments demonstrate that only the (2) construct was used in effective RISC assembly for silencing target EGFR. One mature miRNA, namely miR-EGFP(280/302), was detected in the (2) -transfected zebrafishes, whereas the (1) transfection produced another kind of miRNA, miR*-EGFP(301-281), which is partially complementary to the miR-EGFP(280/ 302). (B) In vivo gene silencing efficacy was only observed in the transfection of the (2) pre-miRNA, while miRNA indicator RGFP (red) was evenly present in all vector transfections. (C) Western blot analysis of the EGFP protein levels confirmed the specific silencing result of (B). No detectable gene silencing was observed in fishes without (Ctl) and with liposome only (Lipo) treatments. The transfection of either a U6-driven siRNA vector (siR) or an empty vector (Vctr) without the designed pre-miRNA insert resulted in no gene silencing significance.

skins demonstrates that this ancient intron-mediated gene regulation system is highly functional in vertebrates. The introns that involved in this regulatory fine-tuning of gene expression is mediated through the activation of miRNA-mediated RNAi effects. Evolutionally speaking, the genome remarkably increases the complexity and variety of introns in higher developed plants and animals, therefore, the influence of the intronic gene regulation to facilitate genome stability and gene coordination is progressively established. Conceivably, dysregulation of the intronic miRNAs is likely to reveal the interwined actions between intronic miRNAs and gene expressions, leading to a better understanding of the genetic etiology of human diseases. The success of intronic miRNA generation by artificial means in vivo may provide a powerful tool to study the mechanism by which of miRNAs induce diseases and potentially shed light on the novel design of gene therapy.

\section{Acknowledgements}

This study was supported by NIH/NCI grant CA-85722. 


\section{References}

1. Lin S.L., Chuong C.M. and Ying S.Y., A Novel mRNAcDNA interference phenomenon for silencing bcl-2 expression in human LNCaP cells. Biochem. Biophys. Res. Commun. 281: 639-644, 2001.

2. Lin S.L., Chang D., Wu D.Y. and Ying S.Y., A novel RNA splicing-mediated gene silencing mechanism potential for genome evolution. Biochem. Biophys. Res. Commun. 310: 754-760, 2003.

3. Ying S.Y. and Lin S.L., Intron-derived microRNAs-fine tuning of gene functions. Gene 342: 25-28, 2004.

4. Clement J.Q., Qian L., Kaplinsky N. and Wilkinson M.F., The stability and fate of a spliced intron from vertebrate cells. RNA 5: 206-220, 1999.

5. Ambros V., Lee R.C., Lavanway A., Williams P.T. and Jewell D., MicroRNAs and other tiny endogenous RNAs in C. elegans. Curr. Biol. 13: 807-818, 2003.

6. Rodriguez A., Griffiths-Jones S., Ashurst J.L. and Bradley A., Identification of mammalian microRNA host genes and transcription units. Genome Res. 14: 1902-1910, 2004.

7. Parrish S., Fleenor J., Xu S., Mello C. and Fire A., Functional anatomy of a dsRNA trigger: differential requirement for the two trigger strands in RNA interference. Mol. Cell 6: 1077-1087, 2000.

8. Holen T., Amarzguioui M., Wiiger M.T., Babaie E. and Prydz H., Positional effects of short interfering RNAs targeting the human coagulation trigger Tissue Factor. Nucl. Acids Res. 30: 1757-1766, 2002.

9. Hutvagner G. and Zamore P.D., A microRNA in a multiple-turnover RNAi enzyme complex. Science 297: 2056-2060, 2002.

10. Zeng Y., Yi R. and Cullen B.R., MicroRNAs and small interfering RNAs can inhibit mRNA expression by similar mechanisms. Proc. Natl. Acad. Sci. USA 100: 9779-9784, 2003.

11. Hall I.M., Shankaranarayana G.D., Noma K., Ayoub N., Cohen A. and Grewal S.I., Establishment and maintenance of a heterochromatin domain. Science 297: 2232-2237, 2002.

12. Llave C., Xie Z., Kasschau K.D. and Carrington J.C., Cleavage of Scarecrow-like mRNA targets directed by a class of Arabidopsis miRNA. Science 297: 2053-2056, 2002.

13. Rhoades M.W., Reinhart B.J., Lim L.P., Burge C.B., Bartel B. and Bartel D.P., Prediction of plant microRNA targets. Cell 110: 513-520, 2002.

14. Lee R.C., Feibaum R.L. and Ambros V., The C. elegans heterochromic gene lin-4 encodes small RNAs with antisense complementarity to lin-14. Cell 75: 843-854, 1993.

15. Reinhart B.J., Slack F.J., Basson M., Pasquinelli A.E., Bettinger J.C., Rougvie A.E., Horvitz H.R. and Ruvkun G., The 21-nucleotide let-7 RNA regulates developmental timing in Caenorhabditis elegans. Nature 403: 901-906, 2000.

16. Lau N.C., Lim L.P., Weinstein E.G. and Bartel D.P., An abundant class of tiny RNAs with probable regulatory roles in Caenorhabditis elegans. Science 294: 858-862, 2001.

17. Brennecke J., Hipfner D.R., Stark A., Russell R.B. and Cohen S.M.., Bantam encodes a developmentally regulated microRNA that controls cell proliferation and regulates the proapoptotic gene hid in Drosophila. Cell 113: 25-36, 2003.

18. Xu P., Vernooy S.Y., Guo M. and Hay B.A., The Drosophila microRNA Mir-14 suppresses cell death and is required for normal fat metabolism. Curr. Biol. 13: 790795, 2003.

19. Lagos-Quintana M., Rauhut R., Meyer J., Borkhardt A. and Tuschl T., New microRNAs from mouse and human. RNA 9: 175-179, 2003.

20. Mourelatos Z., Dostie J., Paushkin S., Sharma A., Charroux B., Abel L., Rappsilber J., Mann M. and Dreyfuss G., miRNPs: a novel class of ribonucleoproteins containing numerous microRNAs. Genes Dev. 16: 720-728, 2002.

21. Zeng Y., Wagner E.J. and Cullen B.R., Both natural and designed micro RNAs can inhibit the expression of cognate mRNAs when expressed in human cells. Mol. Cell 9: 13271333, 2002.

22. Lin S.L., Chuong C.M. and Ying S.Y., D-RNAi (messenger RNA-antisense DNA interference) as a novel defense system against cancer and viral infections. Curr. Cancer Drug Targets 1: 241-247, 2001.

23. Carthew R.W., Gene silencing by double-stranded RNA. Curr. Opin. Cell Biol. 13: 244-248, 2001.

24. Lee Y., Kim M., Han J., Yeom K.H., Lee S., Baek S.H. and Kim V.N., MicroRNA genes are transcribed by RNA polymerase II. EMBO J. 23: 4051-4060, 2004.

25. Lee Y., Ahn C., Han J., Choi H., Kim J., Yim J., Lee J., Provost P., Radmark O., Kim S. and Kim V.N., The nuclear RNase III Drosha initiates microRNA processing. Nature 425: 415-419, 2003.

26. Lund E., Guttinger S., Calado A., Dahlberg J.E. and Kutay U., Nuclear export of microRNA precursors. Science 303: 95-98, 2004.

27. Yi R., Qin Y., Macara I.G. and Cullen B.R., Exportin-5 mediates the nuclear export of pre-microRNAs and short hairpin RNAs. Genes Dev. 17: 3011-3016, 2003.

28. Schwarz D.S., Hutvagner G., Du T., Xu Z., Aronin N. and Zamore P.D., Asymmetry in the assembly of the RNAi enzyme complex. Cell 115: 199-208, 2003.

29. Khvorova A., Reynolds A. and Jayasena S.D., Functional siRNAs and miRNAs exhibit strand bias. Cell 115: 209216, 2003.

30. Lee Y.S., Nakahara K., Pham J.W., Kim K., He Z., Sontheimer E.J. and Carthew R.W., Distinct roles for Drosophila Dicer-1 and Dicer-2 in the siRNA/miRNA silencing pathways. Cell 117: 69-81, 2004.

31. Liquori C.L., Ricker K., Moseley M.L., Jacobsen J.F., Kress W., Naylor S.L., Day J.W. and Ranum L.P.W., Myotinic dystrophy type 2 caused by a CCTG expansion in intron 1 of ZNF9. Science 293: 864-867, 2001.

32. Jin P., Alisch R.S. and Warren S.T., RNA and microRNAs in fragile X mental retardation. Nat Cell Biol. 6: 10481053, 2004.

33. Eberhart D.E., Malter H.E., Feng Y. and Warren S.T., The fragile $\mathrm{X}$ mental retardation protein is a ribonucleoprotein containing both nuclear localization and nuclear export signals. Hum. Mol. Genet. 5: 1083-1091, 1996.

34. Tuschl T. and Borkhardt A., Small interfering RNAs: a revolutionary tool for the analysis of gene function and gene therapy. Mol. Interv. 2: 158-167, 2002.

35. Miyagishi M. and Taira K., U6 promoter-driven siRNAs with four uridine $3^{\prime}$ overhangs efficiently suppress targeted gene expression in mammalian cells. Nat. Biotechnol. 20: 497-500, 2002.

36. Lee N.S., Dohjima T., Bauer G., Li H., Li M.J., Ehsani A., Salvaterra P. and Rossi J., Expression of small interfering RNAs targeted against HIV-1 rev transcripts in human cells. Nat. Biotechnol. 20: 500-505, 2002. 
37. Paul C.P., Good P.D., Winer I. and Engelke D.R., Effective expression of small interfering RNA in human cells. Nat. Biotechnol. 20: 505-508, 2002.

38. Xia H., Mao Q., Paulson H.L. and Davidson B.L., siRNAmediated gene silencing in vitro and in vivo. Nat. Biotechnol. 20: 1006-1010, 2002.

39. McCaffrey A.P., Meuse L., Pham T.T., Conklin D.S., Hannon G.J. and Kay M.A., RNA interference in adult mice. Nature 418: 38-39, 2002.

40. Gunnery S., Ma Y. and Mathews M.B., Termination sequence requirements vary among genes transcribed by RNA polymerase III. J. Mol. Biol. 286: 745-757, 1999.

41. Schramm L. and Hernandez N., Recruitment of RNA polymerase III to its target promoters. Genes Dev. 16: 2593-2620, 2002.

42. Sledz C.A., Holko M., de Veer M.J., Silverman R.H. and Williams B.R., Activation of the interferon system by short-interfering RNAs. Nat. Cell. Biol. 5: 834-839, 2003.

43. Lin S.L. and Ying S.Y., Combinational therapy for HIV-1 eradication and vaccination. Int. J. Oncol. 24: 81-88, 2004.
44. Stark G.R., Kerr I.M., Williams B.R., Silverman R.H. and Schreiber R.D., How cells respond to interferons. Annu. Rev. Biochem. 67: 227-264, 1998.

45. Lin S.L. and Ying S.Y., New drug design for gene therapy Taking Advantage of Introns. Lett. Drug Design Disc. 1: 256-262, 2004.

46. Lin S.L. and Ying S.Y., Novel RNAi therapy - Intronderived microRNA drugs. Drug Design Rev. 1: 247-255, 2004.

47. Nott A., Meislin S.H. and Moore M.J., A quantitative analysis of intron effects on mammalian gene expression. RNA 9: 607-617, 2003.

48. Zhang G., Taneja K.L., Singer R.H. and Green M.R., Localization of pre-mRNA splicing in mammalian nuclei. Nature 372: 809-812, 1994.

49. Ghosh S. and Garcia-Blanco M.A., Coupled in vitro synthesis and splicing of RNA polymerase II transcripts. RNA 6: 1325-1334, 2000.

50. Ying S.Y. and Lin S.L., Intronic microRNAs. Biochem. Biophys. Res. Commun. 326: 515-520, 2005. 